\title{
Pedagogías PARA La CONCIENCIA SOlidaria: MUJER Y CONFLICTO ARMAdo
}

\section{PEDAGOGÍAS FOR THE SOLIDARY CONSCIENCE: WOMAN AND ARMED CONFLICT}

\author{
Sonia Bibiana Rojas Wilches ${ }^{1}$ \\ Claudia Mercedes Jiménez Garcés ${ }^{2}$ \\ Universidad Nacional Abierta y a Distancia, UNAD
}

\section{Resumen}

El presente artículo recoge la experiencia académica de formación para la investigación de estudiantes universitarios en el tema de mujer y conflicto armado como estrategia pedagógica dentro del Curso Sociología de la Universidad Nacional Abierta y a Distancia - UNAD. La reflexión que se presenta atiende a la identificación de problemáticas abordadas en torno al tema y las miradas que se proyectan en su construcción. La importancia de ello, es la conjugación de ejercicios de responsabilidad social universitaria para hacer lecturas de paz en escenarios de post - conflicto.

Palabras clave: mujer, conflicto armado, subjetividad, feminidad, conciencia solidaria.

\section{Abstract}

This paper presents the academic experience of training for research of university students on the theme of women and armed conflict as a teaching strategy in the Sociology course of the Universidad Nacional Abierta y a Distancia- UNAD. Reflection that occurs serves the identification of issues addressed on the issue and looks that are projected in construction. The importance of this is the combination of exercises of university social responsibility to make peace readings scenarios post - conflict.

Keywords: women, armed conflict, subjectivity, femininity, solidarity awareness.

Recibido: 08 de noviembre de 2015

Aceptado: 01 de diciembre de 2015

La construcción de la conciencia solidaria: una obligación a la hora de comprender la relación mujer y conflicto armado

Desde el año 2012 se adelanta en Colombia un proceso de negociación entre el gobierno de Juan Manuel Santos y las FARC-EP, que tiene como objetivo poner fin al conflicto armado con esta organización. Estos acuerdos de paz, desarrollados en La Habana - Cuba, han suscitado opiniones diversas entre la población civil: algunas más bien optimistas, ven en este proceso la posibilidad real de superación de la guerra, mientras una amplia franja de opinión ha ganado terreno en el debate sobre la importancia de suscribir un pacto nacional por la convivencia pacífica, pues considera que el logro de la paz no puede depender de un acuerdo entre dos actores, cuando la violencia se ha insertado en la vida cotidiana de

1 Socióloga. Especialista en Educación, Cultura y Política. Candidata a Magister en Estudios Culturales. Docente e investigadora. Programa de Sociología UNAD. Correo: sobirw@yahoo.es

2 Socióloga. Especialista en Educación, Cultura y Política. Candidata a Magister en Género, Sociedad y Políticas. Docente e Investigadora. Programa de Sociología UNAD. Correo: cmjimenezg@gmail.com 
los colombianos. No obstante, sectores amplios y heterogéneos mantienen percepciones desfavorables y desconfianza frente a las concesiones que se puedan hacer a las FARC.

Entre estos últimos sectores, se ha venido legitimando lo que Fabio López de la Roche (2014) ha concebido como "nacionalismo antifariano", construido por el ex presidente Álvaro Uribe Vélez desde su discurso público, y amplificado por los grandes medios de comunicación, cuestión que ha generado una polarización en el país entre "quienes quieren la paz" y "los que buscan mantener la guerra", lo que constituye hoy uno de los principales obstáculos para la culminación exitosa de los diálogos de La Habana, tanto más cuanto que es la ciudadanía la que debe refrendar los acuerdos allí producidos. Hay entonces una recurrente acusación de "los del lado de la paz", sobre la indolencia e insolidaridad de quienes están en contra de los diálogos para con las víctimas, pues paradójicamente muchos de ellos no han vivido la guerra, no han sido afectados por ella, sus hijos no son los que, de uno u otro bando, han tenido que lidiar con la muerte.

Lo que aquí se percibe es una incapacidad para ponerse en lugar del otro, una indiferencia ante el dolor ajeno, por lo que consideramos necesario trabajar sobre el valor de la solidaridad en los diferentes contextos de socialización de la persona, lo que podría coadyuvar a generar el tejido social necesario para adelantar acciones encaminadas a construir el país del post acuerdo, reduciendo la sensación de desesperanza del universo simbólico de la población. En este marco es posible generar reflexiones sobre el papel que juega la educación para la solidaridad en el contexto universitario, y más específicamente en el de la Universidad Nacional Abierta y a Distancia (UNAD), como institución pública comprometida con una acción solidaria, que centra su razón de ser en el proceso de autoconstrucción del hombre como individuo y como especie, teniendo en cuenta una concepción integral de la persona humana como ser viviente, ser social, histórico y cultural (UNAD, Proyecto Académico Pedagógico Solidario, 2011), lo que implica generar procesos de formación integral que trasciendan lo disciplinar.

En ese campo de formación se incluye el Curso Básico de Sociología, dirigido a estudiantes de los diferentes programas de pregrado de la UNAD, que tiene como propósito contribuir a la formación de su conciencia solidaria, lo que implica fortalecer en ellos una sensibilidad frente a las problemáticas del mundo que los rodea, con el fin de permitir su comprensión y a la vez posibilitar, a través las teorías de la Sociología, un cambio social en su entorno, por lo que el ejercicio pedagógico del curso se sustenta en un proceso de formación de la subjetividad política. Para lograr este objetivo, se desarrolla la estrategia de aprendizaje basado en problemas (APB), en la cual se utiliza el planteamiento de un problema como estímulo para que los estudiantes adquieran y generen nuevo conocimiento (Perdomo, 2014).

Para este periodo académico se ha sugerido el abordaje de problemáticas asociadas al tema de la mujer en el conflicto armado a partir de tres grandes momentos: 1 . De clarificación del ámbito y definición del problema, en donde los estudiantes ubican información secundaria (informes de investigación, bases de datos, artículos noticiosos, documentales), que les permita identificar las formas de violencia que más han afectado a las mujeres, escogiendo la de mayor interés para identificar causas, actores involucrados y consecuencias; 2 . De indagación y análisis de información, en donde los estudiantes se acercan a la cotidianidad de estas mujeres, a través de entrevistas abiertas que les permita una mayor comprensión de estas realidades en sus contextos locales, y 3. De solución a la problemática planteada, en donde el grupo a través su producción escrita hace un balance de las comprensiones colectivas que surgen después del estudio de las dinámicas del conflicto armado colombiano y su afectación a la mujer, a la luz de las teorías sociológicas.

Este es un ejercicio de construcción colaborativa de conocimiento que incluye no sólo a los estudiantes, sino a la red de docentes que acompañan su proceso de aprendizaje, por lo que el trabajo de investigación también es desarrollado por ellos de manera simultánea. En ese sentido, el artículo pone en diálogo el ejercicio investigativo que hemos adelantado como docentes del curso, con respecto a las diferentes 
perspectivas de análisis sobre la mujer y el conflicto armado en Colombia -necesario para orientar el desarrollo de la estrategia de aprendizaje-, con los resultados obtenidos en las actividades adelantadas por los estudiantes en la fase de clarificación del ámbito y definición del problema, desde los aportes que es posible ir vislumbrando hacia la formación de una conciencia solidaria.

\section{Conflicto armado y mujer: perspectivas de análisis desde la mirada de los estudiantes}

Es interesante reconocer que a lo largo de la estrategia pedagógica propuesta en el curso, los estudiantes identifican desde las miradas locales, problemáticas que evidencian una urgencia de estudio desde las diferentes disciplinas de formación de cada uno. Dentro de la emergencia de temas de estudio se encuentran:

- La violación sexual como estrategia de guerra.

- La vinculación de niñas a grupos armados.

- La violencia intrafamiliar como consecuencia del conflicto armado.

La violencia en Colombia no piensa en las mujeres como sobrevivientes.

Siendo la primera la de mayor atención y estudio por parte de los estudiantes. En esta relación hay una referencia de teóricos de la sociología en Colombia, en especial investigadores del conflicto armado como Orlando Fals Borda y Gabriel Restrepo. Además de un acercamiento a la sociología del género en el país.

En las reflexiones de un fenómeno como el conflicto armado, por parte de estudiantes universitarios hay varias miradas que salen a flote y que se enlazan con los imaginarios sociales que frente al conflicto se desprenden: la mujer como principal víctima, las vivencias que frente a las dinámicas de violencia experimentan los estudiantes, y por supuesto, aquellas que representan los medios de comunicación, que para muchos es el único referente sobre el conflicto armado. Pero frente a estos tres escenarios hay puntos de encuentro interesantes, por ejemplo, cómo se comprende la feminidad dentro de las tensiones simbólicas y subjetivas en el marco de la guerra.

En primera instancia, el descubrimiento de la feminidad es una búsqueda de la subjetividad dentro de las construcciones de experiencia individual y colectiva, que se evidencian en la cotidianidad. A ese respecto, los estudiantes reconocen que el conflicto armado modifica los roles de género asignados culturalmente, las configuraciones de familia, y además evidencian las tensiones entre lo público y lo privado que soportan las mujeres. En segunda instancia, la relación mujer y guerra: su vinculación a grupos armados, legales e ilegales.

Para los estudiantes, una mujer en escenarios de guerra, se ve enfrentada a cambio de roles por la muerte, la desaparición o por el mismo proceso desplazamiento. En ese sentido, las problemáticas que se desprenden son: el desarraigo familiar que rompe los anclajes de vida, la vulnerabilidad de la mujer y nińa para la vinculación a grupos armados ilegales, y la asignación de nuevos roles. En muchos de los trabajos académicos adelantados, hay una evidencia de relación entre la violencia y la masculinización de la guerra.

En principio porque la guerra transgrede, cambia las feminidades y subjetividades a través de los lenguajes de violencia simbólica que cargan varios detonantes de ruptura de lo comunitario; es que la guerra marca además unos discursos de lo patriarcal sobre las formas mismas de la violencia, que van mucho más allá del hecho violento, porque contienen unos lenguajes que se profundizan en los sujetos:

La guerra siempre ha sido contada por los hombres; ellos han construido 'la historia oficial' sobre la misma, y es su historia la que hace parte del conocimiento reconocido como tal, del cual las mujeres 
han sido excluidas en virtud de un orden simbólico patriarcal, donde el sujeto del pensamiento y del discurso ha sido un ser masculino (Londoño, 2005. p.68).

Pero las condiciones de una masculinización de la guerra no excluyen la mirada de una subjetividad rebelde, es decir, la posibilidad de transformar las condiciones de crisis en términos de transformación de sus realidades a través de procesos de empoderamiento y de organización social. Algo de lo que Bonder (1998) llama nuevas subjetividades como "la reacción a profundas transformaciones de identidad colectiva, la ruptura de fronteras políticas, surgimiento de nuevos sujetos sociales y nuevos conglomerados supranacionales que han socavado los emblemas identitarios nacionales" (p.14).

Otro de los escenarios de estudio de los estudiantes es la identificación del cuerpo de las mujeres como lector de las violencias. En éste punto se presentan varias miradas sobre el cuerpo de la mujer: bien como trofeo de guerra, como estrategia de dominación colectiva, o como lugar de violencias en temas como el uso de anticonceptivos y el aborto obligatorio, por ejemplo, cuando estas mujeres hacen parte de las guerrillas. El despertar de ésta concepción en sus estudios los ha empujado a reflexionar sobre esas otras miradas del conflicto y a interrogarse sobre nuevas concepciones del cuerpo, que entre otras cosas equivale a comprenderlo desde la crisis y el terror. Para Le Breton (2002) un cuerpo en crisis despierta las formas de confrontarse con su realidad pero también de transformarla.

Seguramente esta noción de cuerpo, que los estudiantes han encontrado en sus reflexiones se relaciona con lo expuesto por Consuelo Pabón en su texto Construcciones de Cuerpos: "Todo esto para preguntar por lo que pueden nuestros cuerpos aquí y ahora, en este país, en esta guerra, en este acontecimiento que nos ha tocado vivir" (p.1). El conflicto armado colombiano es transeúnte de los cuerpos y a la vez los transforma: "en el recinto del cuerpo se despliegan simbólicamente los desafíos sociales y culturales" (Le Breton, 19922. p.73).

La guerra y las lecturas de su tránsito sobre las subjetividades, permiten no solo comprender el impacto diferencial de la violencia, sino además de las formas de sufrir, de resistir y de transformar. Según el documento del Centro de Memoria Histórica Mujeres y Guerra, hay una necesidad de estudio: La ocurrencia de la violencia contra la mujer, ya sea en tiempos de paz o en tiempos de guerra, ha sido un asunto soslayado o en el mejor de los casos minimizado (p.18). Es la guerra entonces, como fenómenos social lo que permite hacer lectura de las formas de poder y como tal del uso de la fuerza. La guerra pone en juego la capacidad de resistencia y también las habilidades de transformación de los sujetos.

\section{Conclusiones}

Aportes del proceso formativo a la configuración de una conciencia solidaria: primeras miradlas

En los ejercicios de educación a distancia y mediada a través de las tecnologías, se reconoce la importancia de dos escenarios de aprendizaje: los trabajos colaborativos y los contextos situados de los estudiantes. Sobre estos dos escenarios, hay una suerte de aprendizajes interesantes. El primero sobre la capacidad de lectura de los contextos que los estudiantes logran tener, y el segundo, la capacidad de escucha y de diálogo frente a problemáticas que se presentan de formas particulares, les permite tener una descripción interesante del conflicto armado en Colombia.

Los estudiantes que provienen de diferentes carreras profesionales y que convergen en el Curso de Sociología, descubren e interpretan otras formas de comprender su realidad nacional. Situarse frente a la problemática como ciudadanos, por ejemplo, los lleva a tener más sensibilidad y conciencia frente al conflicto armado, no solo para su explicación posible sino para buscar soluciones y configurar escenarios de participación, lo que coadyuva a la formación de su conciencia solidaria. 
Para Miguel Ramón, esta conciencia surge cuando se comparte la carga y el dolor de los demás, cuando se expresa la sensibilidad social frente al sufrimiento ajeno y se asume como propio; cuando se actúa para superar las barreras sociales que disminuyen a las personas, coartan la libertad y niegan la creatividad, la equidad y la participación social (UNAD, Proyecto Académico Pedagógico Solidario, 2011). Entendemos que el conocimiento de la realidad se asume como condición básica para generar procesos de concientización, que llevan a su vez a la toma de posición, y de allí a la acción transformadora, lo que le permite a los estudiantes el despliegue de su subjetividad política, al plantear alternativas de solución a los problemas que abordan desde la estrategia de aprendizaje.

Es así como la investigación que desarrollan sobre los marcos del conflicto y sus afectaciones a la mujer en Colombia, les ha permitido conocer una realidad que en muchos casos les es ajena, bien sea por su condición de género -la de ser hombres por ejemplo-, su ubicación geográfica, o sus historias de vida particulares. La narración extraída de uno de los foros de trabajo colaborativo, evidencia la transformación en la mirada de una estudiante con respecto al tema abordado:

Sobre los efectos del conflicto en la mujer, solo sabía lo que mostraban los noticieros, sentía tristeza por ver a esa madres sufriendo, pero aun siendo mujer, me parecía una realidad muy lejana. Ahora veo desde otra perspectiva esta situación, porque la sociología te ayuda a ver la realidad de una manera más objetiva, más contextualizada. Sugey Daza Curiel, estudiante de Licenciatura en Inglés.

Asimismo, el ejercicio les ha permitido deconstruir las visiones estereotipadas sobre la mujer, que la ubican en condición de vulnerabilidad, cuestión ligada a la idea de "sexo débil". A ese respecto, vale la pena resaltar cómo en algunos grupos colaborativos, la definición del problema no solo se volcó hacia identificar a la mujer víctima del conflicto, sino a aquella mujer capaz de movilizarse a través de su acción organizada con otras y otros, enfrentando las situaciones problemáticas que la afectan, lo que permitió visibilizar en sus investigaciones, las acciones solidarias que han generado estas mujeres en casos como el de las madres del Caguán, o las madres de Soacha.

\section{Referencias}

Amnistía Internacional (2004). Colombia Cuerpos marcados, crimenes silenciados. Madrid: EDAI

Bonder, G. (1998) Género y Subjetividad: Avatares de una relación no evidente. Programa Interdisciplinario de Estudios de Género (PIEG): Universidad de Chile.

Centro de Memoria Histórica (2011). Mujeres y Guerra. Victimas y resistentes en el Caribe Colombiano. Colombia: Taurus Pensamiento.

Le Breton, D. (1992) Sociología del Cuerpo. Buenos Aires: Ediciones Buena Visión.

Le Breton, D. (2002) Antropología del Cuerpo y Modernidad. Buenos Aires: Ediciones Nueva Visión.

Londońo, L. (2005) La corporalidad de las guerras: una mirada sobre las mujeres combatientes desde el cuerpo y el lenguaje. Revista de Estudios Sociales No. 21. Pp. $67-74$.

López de la Roche, F. (2014). Las ficciones del poder. Patriotismo, medios de comunicación y reorientación afectiva de los colombianos bajo Uribe Vélez (2002-2010). Bogotá: IPEPRI, Universidad Nacional de Colombia.

Pabón, C. (2002). Construcciones de cuerpos. En: Grupo de derechos humanos (Comp.), Expresión y vida: prácticas en la diferencia, Bogotá, Escuela Superior de Administración Pública -ESAP, pp. 36-79.

Perdomo, H. (2014). Syllabus del Curso de Sociología. Unidad Académica Sociohumanística, Universidad Nacional Abierta y a Distancia.

Universidad Nacional Abierta y a Distancia. (2011). Proyecto académico pedagógico solidario. Bogotá, UNAD. Recuperado el 12 de junio de 2015 de http://academia.unad.edu.co/images/pap-solidario/PAP\%20solidario\%20v3.pdf 